\title{
Endovascular Treatment Options of Acute Limb Ischemia
}

\author{
Di Zhang, Wensheng Lou, Guoping Chen, Xindao Yin, Jianping Gu \\ Department of Radiation Physics, Nanjing Hospital, Nanjing Medical University, Nanjing, China \\ Email: yonzhang@mdanderson.org, songgao@mdanderson.org
}

Received October 14, 2012; revised November 15, 2012; accepted November 22, 2012

\begin{abstract}
Acute limb ischemia is an urgent condition which occurs when there is an abrupt interruption of blood flow into an extremity usually because of either embolic or thrombotic vascular occlusion. Restoration of perfusion through early intervention can decrease amputation and mortality. Contemporary treatment includes both surgery and endovascular techniques. There is a rapid progress in endovascular intervention therapy. This article aims to make a comprehensive review of the endovascular intervention options of acute limb ischemia.
\end{abstract}

Keywords: Acute Limb Ischemia; Arterial Embolus; Arterial Thrombosis; Endovascular Therapy

\section{Introduction}

Acute limb ischemia (ALI) of the lower extremities remains a challenging clinical dilemma, which occurs when there is an abrupt interruption of blood flow into an extremity usually because of either embolic or thrombotic vascular occlusion $[1,2]$. The major clinical sign of ALI is "6P", i.e., pain, parasthesia, paralysis, pulselessness, pallor and poikilothermia [3]. The overall prognosis is poor, the mortality rate was $5.3 \%$ - $12 \%$, amputation rate was $5.3 \%-14 \%[1,4-6]$. When profound ischemia ensues, this represents an emergency in which restoration of perfusion through early intervention can lead to limb salvage, whereas delay may result in significant morbidity, including limb loss and, potentially, death. Therefore, timely and correctly treatment of this disease is important.

There are three management options in acute lower limb ischemia: 1) clot removal by catheter-directed thrombolysis with or without percutaneous mechanical thrombectomy; 2) surgical thromboembolectomy followed by correction of underlying arterial lesions; and 3) anticoagulation with continued observation. We can categorize the level of the patient's limb ischemia utilizing clinical assessment of motor and sensory function and interrogating ankle arterial flow velocity signals into several levels [7]. As shown in Table 1, those with level I ischemia, particularly if they have significant comorbidities, can and should be treated with heparin and observation, being watched closely while attention is given to treating associated comorbidities. Conversely, in patients with the same level of ischemia (I), i.e., active patients without significant comorbidities, it is practical to proceed directly with endovascular revascularization
(CDT and, possibly, percutaneous mechanical thrombectomy). This same management choice is equally appropriate for those at level IIA, and at both of these levels of ischemia there should be sufficient time for restoration of patency using either endovascular or open surgical techniques [8]. In the past, the decisional breakpoint in choosing between endovascular and surgical revascularization came between class IIA and class IIB, Treatment of ALI has shifted toward endovascular therapies because of rapidly improving technology and delivery systems unless there is a contraindication, such as profound critical limb ischemia, renal dysfunction, or contrast allergy. Regardless of which kind of therapy to be chosen, the patient should be immediately anticoagulated to prevent further clot extension. This article aims to make a comprehensive review of the endovascular options with intra-arterial thrombolysis and/or adjuvant endovascular techniques.

\section{Catheter Directed Thrombolysis (CDT)}

The development of effective medications to dissolve occluding thrombus led to the increasing use of thrombolytic therapy in patients with ALI. Randomized control trials have provided a rationale for thrombolysis as a first step in patients with ALI vs immediate operative revascularization. Catheter directed thrombolysis offers several potential advantages to lessen the pitfalls of open techniques. By utilizing an endovascular approach and local anesthesia, the risks of general anesthesia are minimized within a subgroup of patients that are at their physiologic limits [9]. In addition, enzymatic dissolution of thrombus allows for more effective clot resolution particularly within distal arterial beds that are often re- 
Table 1. Clinical categories of acute limb ischemia.

\begin{tabular}{|c|c|c|c|c|c|}
\hline Category & Prognosis & Sensory Loss & Motor Deficit & $\begin{array}{l}\text { Arterial } \\
\text { Doppler }\end{array}$ & $\begin{array}{l}\text { Venous } \\
\text { Doppler }\end{array}$ \\
\hline I: Viable & No immediate threat & None & None & Audible & Audible \\
\hline IIA: Marginally threatened & Salvageable if promptly treated & Minimal (toes) or none & None & Inaudible & Audible \\
\hline IIB: Immediately threatened & $\begin{array}{l}\text { Salvageable if immediately } \\
\text { revascularized }\end{array}$ & More than toes, rest pain & Mild/Moderate & Inaudible & Audible \\
\hline III: Irreversible & $\begin{array}{c}\text { Major tissue loss, permanent nerve } \\
\text { damage inevitable }\end{array}$ & $\begin{array}{l}\text { Profound, } \\
\text { anesthetic }\end{array}$ & $\begin{array}{c}\text { Profound, } \\
\text { paralysis }\end{array}$ & Inaudible & Inaudible \\
\hline
\end{tabular}

sistant to open thrombectomy.

There are many evidences from randomized trials comparing catheter-directed thrombolysis with operative revascularization. Ouriel et al. published a study that has come to be known as the Rochester trial. The investigators randomized 114 patients with acute limb ischemia of fewer than 7 days duration to urokinase or surgical intervention. At 1 year, the cumulative risk of amputation $(18 \%)$ was identical in the two groups, while the cumulative survival rate was significantly improved in patients randomized to the thrombolysis group $(84 \%$ vs $58 \%$ at 12 months, $p=0.01)$. The mortality differences seemed to be primarily attributable to an increased frequency of in-hospital cardiopulmonary complications in the operative treatment group $(49 \%$ vs $16 \%, p=0.001)$. The benefits of thrombolysis were achieved without significant differences in the duration of hospitalization (median 11 days) and with only modest increases in hospital cost in the thrombolytic treatment arm (median $\$ 15,672$ vs $\$ 12,253, p=0.02$ ). Thrombolysis was equally effective in those with embolic and thrombotic occlusions, although the survival benefit was greater for patients with embolic occlusions [10]. The Thrombolysis or Peripheral Arterial Surgery (TOPAS) investigators randomized 213 patients with acute lower extremity ischemia secondary to native arterial or bypass graft occlusion of fewer than 14 days duration to a variable dose of recombinant urokinase (rUK) or surgery. Among patients treated with rUK, surgical operations were avoided in $46 \%$ of patients and the magnitude of such procedures was reduced in $50 \%$ of cases. Survival and amputation-free survival at 12 months were similar in the rUK and surgical groups. Amputation-free survival was similar in the two groups. There was a trend toward a higher amputation-free survival among those randomized to surgery and significantly more bleeding in those randomized to rUK. Among patients treated with rUK, thrombus resolution and clinical outcome were somewhat better for acute bypass graft thrombosis than for native arterial occlusion. For thrombi longer than $30 \mathrm{~cm}$, post-hoc analysis suggested that 1-year amputation-free survival was better following thrombolytic treatment, potentially due to lack of suitable outflow for a surgical procedure. In contrast, shorter occlusions fared better with surgery [11]. Although The Surgery versus Thrombolysis for Ischemia of the Lower Extremity (STILE) trial was prematurely terminated by the Data and Safety Monitoring Board, subsequent analysis, however, offered important insight. Patients presenting with acute ischemia (14 or fewer days) and randomized to thrombolysis had significantly better limb salvage ( $89 \%$ vs $70 \%$ ) and amputation-free survival [12].

It is very important to monitor coagulation parameters carefully which aim to reduce risk hemorrhagic complications (estimated at $6 \%$ to $12.5 \%$ ) during CDT procedure [3,9-11]. Fibrinogen levels should also be checked as a level $<100 \mathrm{mg} / \mathrm{dL}$ which reflects systemic fibrinolysis and an increased risk of bleeding [12].

The severity of acute limb ischemia should be considered as the basis for therapic decision if we could use CDT. CDT has long re-flow time, which can aggravate ischemic. ALI of a limb artery can occur in the presence or absence of underlying atherosclerotic involvement. If there is no underlying atherosclerotic disease of the limb, the thromboembolic event usually presents with profoundly ischemic lower extremity, whereas thromboembolic occlusion of chronically diseased lower limb artery may present only with mild progression of chronic symptoms because of the development of collateral vessels [8]. If ALI is located in the chronically diseased artery, CDT could be the first choice.

We should master contraindications for CDT in ALI in case of fatal haemorrhage $[2,13]$. Absolute contraindications to thrombolysis are well-known and include active bleeding, central nervous system injury, or major operative procedures within the preceding 2 weeks. Relative contraindications include uncontrolled hypertension, recent eye surgery, pregnancy, and intracranial neoplasms.

\section{Percutaneous Mechanical Thrombectomy (PMT)}

CDT is associated with slow restoration of blood flow, which may aggravate tissue damage. The advent of PMT has allowed removing clot burden quickly with CDT bolstered by using of PMT devices and can be used in pa- 
tients with acute profoundly limb ischemia. The earlier clot-removing methods are applied, the better the outcomes. Although no randomized prospective study showed that safety and efficacy of percutaneous mechanical thrombectomy or combined with thrombolysis in the treatment of ALI, some data has shown its advantage in fastreflow and improvement of patients' symptoms, and PMT may be the only available treatment option in patients at high risk for open surgery or with contraindications to pharmacologic thrombolysis. K. Kasirajan et al. published that thrombus removal rate was $85 \%$, using a PMT catheter as an initial treatment for acute $(<2$ weeks $)$ and subacute ( 2 weeks to 4 months) arterial occlusion of the limbs. Angiographic outcome was not dependent on the duration of occlusion or the conduit type [14]. Ansel GM and his colleagues showed results of treatment of acute limb ischemia with a percutaneous mechanical thrombectomy-based endovascular approach. Angiography following PMT showed thrombus removal complete/ substantial $63.6 \%$, partial $28.0 \%$, and minimal $8.8 \%$, respectively, and amputation free survival of acute limb ischemia treated with PMT alone or in combination with thrombolysis was $94.7 \%$ at five-year follow-up [15]. Oguzkurt L et al. reported that complete thrombus removal with PMT was achieved in $90 \%$ with acute occlusions. Amputation-free survival rate was $100 \%$ at one month, $93 \%$ at one year, and $93 \%$ at two years [16].

There is a new effective method to dissolve occluding thrombus reported recently which using isolated pharmaco-mechanical thrombolysis-thrombectomy (IPMT) to isolate the thrombus between two balloons and utilize wire oscillation to increase the thombus-lytic exposure surface area followed by aspiration. IPMT decreases systemic lytic exposure, procedure time, and distal embolism [17].

PMT has generally been used as an adjunctive method to CDT, but reverse was also true, and CDT could be an adjunctive to PMT. Total dose of thrombolytic drug used was low because PMT removed most of the thrombi in most cases [14,15]. Main disadvantages of PMT were the need for larger vascular sheath for large guiding catheter than usual and the dissection of the artery. It should be noted that a patient's symptoms may get transiently worse as the thrombus fragments with distal emboli. Distal embolisation has been reported in $10 \%$ of cases with the PMT procedure [16]. So during PMT, the operator should perform gently to reduce risk of distal embolisation and vascular injury.

\section{Angioplasty}

Successful clot dissolution will unveil a "culprit" lesion responsible for initiating thrombosis. Angioplasty is not the preferred initial method of treatment for ALI, only in patients considered unfit for thrombolysis or in patients who had undergone unsuccessful surgical recanalisation or treatment with residual stenosis, as well as dealing with intraoperative complications remedies [18]. Arteriography following removal of the thrombus by thrombolysis, and/or PMT usually delineates the responsible lesion and, a decision must then be made in regard to how best to manage this lesion and eliminate the threat of recurrence it poses. Discrete atherosclerotic lesions are well managed by either balloon angioplasty or, if accessible in the surgical field, by surgical revision with patch angioplasty, both yielding durable results. However, longer, more extensive lesions or multiple stenoses-inseries are still best treated by bypass. Kashyap et al. reported in an overwhelming majority of cases (91\%), an adjuvant procedure was performed to treat the "culprit lesion" leading to thrombosis. This was a purely percutaneous endovascular procedure in 56\%, an open surgical procedure in $15 \%$ and a combined approach in $28 \%$ [4]. Plate et al. [19] reported that angioplasty ratio after CDT and/or PMT was $68 \%$; PTA was $60 \%, 5 \%$ stent implantation, aneurysm exclusion $2 \%$, and $1 \%$ endarterectomy. Kim et al. reported 15 cases of ALI underwent stent implantation, technical success rate was $100 \%$. Duration of the intervention ranged between $20 \mathrm{~min}$ and $50 \mathrm{~min}$. During the average follow-up of 14.4 months, no re-occlusion or restenosis was observed by CT angiography or duplex USG [18]. Oguzkurt et al. [16] reported using long-duration balloon inflation with/ without stent placement to fix artery dissection. The articles about stenting in ALI gradual increase in recent years, with good clinical efficacy and no occurrence of distal embolization [20, 21]. Stent-assisted recanalisation may be an effective treatment for patients with acute limb ischaemia who are considered unfit for thrombolysis or surgical recanalisation or who have severe ischaemia requiring immediate recanalisation or occlusion in the iliac arteries. Berczi et al. [22] treated seven acute thrombotic occlusions in the iliac arteries by primary stent implantation without distal embolisation.

\section{Embolic Protection Devices (EPDs)}

Distal embolization following percutaneous revascularization procedures is a universal phenomenon that has been reported in various vascular beds, including the carotid and renal arteries and the lower extremities. Distal embolization may make it necessitate the use of additional interventions, including thrombectomy or thrombolysis, resulting in longer procedure time, greater amount of contrast used, and larger radiation exposure. EPDs are considered as a "standard of care", during percutaneous carotid interventions, coronary artery bypass graft PCI, and even renal angioplasty based on recent data. Although the FDA has not ratified any EPDs for peripheral arterial, several works have shown the safety and 
feasibility of EPDs use in this vascular territory. There was a report, the first time in 2003, about using EPDs in peripheral vascular angioplasty, and filter membranes were evaluated with scanning electron microscopy. System delivery was successful in all patients [23]. D. Siablis et al. [24] investigated the feasibility, safety and distal emboli protection capability during recanalization of lower extremities' acute and subacute occlusions. Technical success rate of deployment and utilization of the filtration devices was $100 \%$. Macroscopic particulate debris was extracted from all the filters containing fresh thrombus, calcification minerals, cholesterol and fibrin. Shammas et al. [25] reported that there were no complications related to the release and recovery process of the device. Although many researchers believe patients with high risk for distal embolic, poor outflow tract and direct plaque excision will derive the greatest benefit from the use of this technology, large multicenter registry is needed to define the exact effects for these devices, to improve the technical design for this unique vascular bed, and clearly to identify best indications [23-25].

\section{Summary}

The treatment methods chosen should be based on the patient's conditions and angiographic results during clinical practice. Now more and more interventional therapies for ALI which derive from combinations of several procedures above are used to restore blood flow quickly and reduce complications. CDT combined with PMT plus PTA/stent for ALI can be effective and minimally invasive. In the future, one can expect to improve endovascular techniques to the point where it will more significantly impact on the management of acute as well as chronic lower extremity occlusive diseases.

\section{REFERENCES}

[1] J. L. ELiason, R. M. Wainess, M. C. Proctor, J. B. Dimick, J. A. Cowan Jr., et al., "A National and Single Institutional Experience in the Contemporary Treatment of Acute Lower Extremity Ischemia," Annals of Surgery, Vol. 238, No. 3, 2003, pp. 382-390.

[2] C. Kyriakides, W. G. Austen Jr., Y. Wang, J. Favuzza, F. D. Moore Jr., et al., "Neutrophil Mediated Remote Organ Injury after Lower Torso Ischemia and Reperfusion Is Selectin and Complement Dependent," The Journal of Trauma, Vol. 48, No. 1, 2000, pp. 32-38. doi:10.1097/00005373-200001000-00006

[3] M. Dvorak, M. Vlasin, M. Dvorakova, P. Rauser and L. Lexmaulova, "Heparin and Its Derivatives in the Treatment of Arterial Thrombosis: A Review," Veterinarni Medicina, Vol. 55, No. 11, 2010, pp. 523-546.

[4] V. S. Kashyap, R. Gilani, J. F. Bena, M. Bannazadeh and T. P. Sarac, "Endovascular Therapy for Acute Limb Ischemia," Journal of Vascular Surgery, Vol. 53, No. 2, 2011,

\section{pp. 340-346. doi:10.1016/j.jvs.2010.08.064}

[5] Z. Mitrev, F. Beyersdorf, R. Hallmann, Y. Poloczek, K. Ihnken, et al., "Reperfusion Injury in Skeletal Muscle: Controlled Limb Reperfusion Reduces Local and Systemic Complications after Prolonged Ischaemia," Cardiovascular Surgery, Vol. 2, No. 6, 1994, pp. 737-748.

[6] K. Kasirajan, F. P. Beavers, D. G. Clair, D. G. Clair, R. Greenberg, et al., "Rheolytic Thrombectomy in the Management of Acute and Subacute Limb Threatening Ischemia," Journal of Vascular and Interventional Radiology, Vol. 12, No. 4, 2001, pp. 413-420. doi:10.1016/S1051-0443(07)61878-8

[7] R. B. Rutherford, J. D. Bake, C. Ernst, et al., Recommended Standards for Reports Dealing with Lower Extremity Ischemia: Revised Version," Journal of Vascular Surgery, Vol. 26, No. 3, 1997, pp. 517-538. doi:10.1016/S0741-5214(97)70045-4

[8] R. B. Rutherford, "Clinical Staging of Acute Limb Ischemia as the Basis for Choice of Revascularization Method: When and How to Intervene," Seminars in Vascular Surgery, Vol. 22, No. 1, 2009, pp. 5-9. doi:10.1053/j.semvascsurg.2008.12.003

[9] K. Ouriel and F. J. Veith, "Acute Lower Limb Isehemia: Determinants of Outcome," Surgery, Vol. 124, No. 4, 1998, pp. 336-342. doi:10.1016/S0039-6060(98)70139-4

[10] K. Ouriel, C. K. Shortell, J. A. DeWeese, et al., "A comparison of Thrombolytic Therapy with Operative Revascularization in the Initial Treatment of Acute Peripheral Arterial Ischemia," Journal of Vascular Surgery, Vol. 19, No. 6, 1994, pp. 1021-1030. doi:10.1016/S0741-5214(94)70214-4

[11] K. Ouriel, F. J. Veith and A. A. Sasahara, "A Comparison of Recombinant Urokinase with Vascular Surgery as Initial Treatment for Acute Arterial Occlusion of the Legs. Thrombolysis or Peripheral Arterial Surgery (TOPAS) Investigators," The New England Journal of Medicine Vol. 338, No. 16, 1998, pp. 1105-1111. doi:10.1056/NEJM199804163381603

[12] The STILE Investigators, "Results of a Prospective Randomized Trial Evaluating Surgery versus Thrombolysis for Ischemia of the Lower Extremity. The STILE Trial: The STILE Investigators (Appendix A)," Annals of Surgery, Vol. 220, No. 3, 1994, pp. 251-268. doi:10.1097/00000658-199409000-00003

[13] A. S. Ward, S. K. Andaz and S. Bygrave, "Thrombolysis with Tissue-Plasminogen Activator: Results with a HighDose Transthrombus Technique," Journal of Vascular Surgery, Vol. 19, No. 3, 1994, pp. 503-508. doi:10.1016/S0741-5214(94)70078-8

[14] K. Kasirajan, F. P. Beavers, D. G. Clair, R. Greenberg, et al., "Rheolytic Thrombectomy in the Management of Acute and Subacute Limb Threatening Ischemia," Journal of Vascular and Interventional Radiology, Vol. 12, No. 4, 2001, pp. 413-420. doi:10.1016/S1051-0443(07)61878-8

[15] G. M. Ansel, C. F. Botti and M. J. Silver, "Treatment of Acute Limb Ischemia with a Percutaneous Mechanical Thrombectomy-Based Endovascular Approach: 5-Year Limb Salvage and Survival Results from a Single Center 
Series," Catheterization and Cardiovascular Interventions, Vol. 72, No. 3, 2008, pp. 325-330. doi: $10.1002 / \mathrm{ccd} .21641$

[16] L. Oguzkurt, U. Ozkan, B. Gumus, I. Coşkun, N. Koca, et al., "Percutaneous Aspiration Thrombectomy in the Treatment of Lower Extremity Thromboembolic Occlusions," Diagnostic and Interventional Radiology Diagnostic, Vol. 16, No. 1, 2010, pp. 79-83.

[17] R. Gupta and T. A. Hennebry, "Percutaneous Isolated Pharmaco-Mechanical Thrombolysis-Thrombectomy System for the Management of Acute Arterial Limb Ischemia: 30-Day Results from a Single-Center Experience," Catheterization and Cardiovascular Interventions, Vol. 80, No. 4, 2012, pp. 636-643. doi:10.1002/ccd.24283

[18] C. Kim, W. Jeon, T. Shin, D. Choi, J. Kim, et al., "StentAssisted Recanalisation of Acute Occlusive Arteries in Patients with Acute Limb Ischaemia," European Journal of Vascular and Endovascular Surgery, Vol. 39, No. 1, 2010, pp. 89-96. doi:10.1016/j.ejvs.2009.09.023

[19] G. Plate, I. Jansson, C. Forssell, P. Weber and S. Oredsson, "Thrombolysis for Acute Lower Limb Ischaemia-A Prospective, Randomised, Multicentre Study Comparing Two Strategies," European Journal of Vascular and Endovascular Surgery, Vol. 31, No. 6, 2006, pp. 651-660. doi:10.1016/j.ejvs.2005.11.017

[20] J. Raja, G. Munneke, R. Morgan and A. M. Belli, "Stenting in Acute Lower Limb Arterial Occlusions," Cardiovascular and Interventional Radiology, Vol. 31, No. S2, 2008, pp. S41-S44. doi:10.1007/s00270-007-9052-3
[21] S. Yilmaz, T. Sindel and E. Lüleci, "Primary Stenting of Embolic Occlusions in Iliac Arteries," Journal of Endovascular Therapy, Vol. 10, No. 3, 2003, pp. 629-635. doi:10.1583/1545-1550(2003)010<0629:PSOEOI $>2.0 . \mathrm{C}$ $\underline{\mathrm{O} ; 2}$

[22] V. Berczi, S. M. Thomas, D. R. Turner, J. R. Bottomley, T. J. Cleveland, et al., "Stent Implantation for Acute Iliac Artery Occlusions: Initial Experience," Journal of Vascular and Interventional Radiology, Vol. 17, No. 4, 2006, pp. 645-649. doi:10.1097/01.RVI.0000203918.91835.73

[23] C. W. König, B. Pusich, G. Tepe, H. P. Wendel, U. Hahn, et al., "Frequent Embolization in Peripheral Angioplasty: Detection with an Embolism Protection Device (Angioguard) and Electron Microscopy," Cardiovascular and Interventional Radiology, Vol. 26, No. 4, 2003, pp. 334 349. doi:10.1007/s00270-003-2656-3

[24] D. Siablis, D. Karnabatidis, K. Katsanos, P. Ravazoula, P. Kraniotis, et al., "Outflow Protection Filters during Percutaneous Recanalization of Lower Extremities' Arterialocclusions: A Pilot Study," European Journal of Radiology, Vol. 55, No. 2, 2005, pp. 243-249. doi:10.1016/j.ejrad.2004.07.010

[25] N. W. Shammas, E. J. Dippel, D. Coiner, G. A. Shammas, M. Jerin, et al., "Preventing Lower Extremity Distal Embolization Using Embolic Filter Protection: Results of the PROTECT Registry," Journal of Endovascular Therapy, Vol. 15, No. 3, 2008, pp. 270-276. doi:10.1583/08-2397.1 